- General medical practitioners' awareness of oral cancer risk factors and clinical appearance is less than that of their dental counterparts.

- Patients can present to their general medical practitioners with oral lesions.

- Alcohol use must be emphasised as a risk factor for oral cancer in future training of health professionals.

- The significance of erythroplakia and erythroleukoplakia needs to be emphasised in future training of health professionals.

- This paper highlights the need for improved education of general medical and general dental practitioners on oral cancer.

\title{
Oral cancer awareness of general medical and general dental practitioners
}

\author{
L. M. Carter ${ }^{1}$ and G. R. Ogden ${ }^{2}$
}

\author{
Objective To assess general medical practitioners' (GMPs') and \\ general dental practitioners' (GDPs') awareness of prevention and early \\ detection of oral cancer. \\ Design Cross-sectional questionnaire-based study. \\ Setting Primary care. \\ Method Questionnaires were delivered to all GMPs and GDPs in \\ Tayside, assessing oral examination habits, delivery of advice on oral \\ cancer risk factors, knowledge of oral cancer risk factors and clini- \\ cal appearance, preferred point of referral and requests for further \\ information.
}

Results Response rates of 71\% and 84\% were obtained from GMPs and GDPs respectively. GMPs were less likely to examine patients' oral mucosa routinely, less likely to advise patients about risk factors for oral cancer, identified fewer risk factors for, and felt less confident about diagnosing oral cancer from clinical appearance than their dental counterparts. Seventy-one percent and $80 \%$ of GMPs and GDPs respectively requested further information about oral cancer.

Conclusion Patients with oral lesions often present to their general medical practitioner. The incidence of oral cancer is rising in the United Kingdom, therefore the role of general medical practitioners in prevention and detection of oral cancer is becoming ever more important. This study highlights the need for improved education of general medical practitioners on oral cancer.

\section{INTRODUCTION}

The incidence of oral cancer is increasing in the United Kingdom. ${ }^{1-3}$ In the UK the incidence of oral cancer is greater than that of cervical cancer ${ }^{4}$ and the incidence of oral cancer in men

\footnotetext{
"Specialist Registrar, Oral and Maxillofacial Surgery, Leeds Dental Institute, Clarendon Way, Leeds, LS2 9LU; ${ }^{2}$ Professor of Oral and Maxillofacial Surgery, Dundee Dental Hospital and School, Park Place, Dundee, Tayside, DD1 4NP

*Correspondence to: Dr Lachlan M. Carter

Email: lachlan_carter@hotmail.com
}

Online article number E10

Refereed Paper - accepted 5 March 2007

DOI: $10.1038 /$ bdj.2007.630

${ }^{\circledR}$ British Dental Journal 2007; 203: E10 in Scotland is similar to that of cervical cancer. Incidence rates are increasing in women and younger patients. ${ }^{3,5}$

Ninety-five percent of oral cancers are squamous cell carcinomas. Many of these malignancies have avoidable aetiological risk factors. ${ }^{6}$ The major risk factors in the UK are tobacco and alcohol use. Early detection of oral cancers makes them more amenable to treatment, thus reducing morbidity and allowing the greatest chance of cure. ${ }^{6,7}$ Whilst public awareness of oral cancer has been shown to be poor, there is some evidence that certain groups may be more aware. ${ }^{8,9}$ More people may have heard of mouth cancer thanks to initiatives like Mouth Cancer Awareness Week (MCAW) or the West of Scotland Cancer Awareness Project (WoSCAP). However, the increased publicity from Mouth Cancer Awareness Week has not led to a reduction in patient or referral delay ${ }^{10}$ and risk factor knowledge ${ }^{11}$ and awareness of the signs and symptoms of the disease are still poor. ${ }^{8,9,12}$

Lack of public awareness has been reported in the past to be the most significant factor in delaying referral and treatment of oral cancer, ${ }^{10,13}$ although ignorance of early signs is probably the most important. Lack of general medical practitioner (GMP) and general dental practitioner (GDP) knowledge has also been shown to contribute to delays in referral and treatment. ${ }^{14}$ A good deal of literature exists on GDP oral cancer awareness, however little is known about GMP oral cancer awareness in the United Kingdom. ${ }^{15}$ As GMPs and GDPs refer similar proportions of patients to maxillofacial units, ${ }^{14,16}$ and as patients often consult their GMP rather than their GDP with oral lesions, ${ }^{17-19}$ the aim of this study was to assess GMP as well as GDP oral cancer awareness.

\section{METHOD}

A questionnaire was sent to all GMPs (334) and GDPs (157) in Tayside (Fig. 1). Ten questions were asked, investigating: oral cancer screening/oral mucosal examination habits; knowledge and delivery of advice on risk factors for oral cancer; knowledge and confidence regarding appearance of oral changes associated with oral cancer; point of referral selection; and 
opinions on sufficiency of individual knowledge on oral cancer detection and prevention, desire for further information/training and the format of such information/training. The questionnaire required approximately ten minutes to complete. A repeat mailing was undertaken for non-responders. The results were analysed using the Student's t-test, $\chi^{2}$ and extended $\chi$ tests.

\section{RESULTS}

Questionnaires were returned by 238 GMPs and by 133 GDPs, producing return rates of $71.26 \%$ and $84.71 \%$ respectively. Significantly more GDPs (95.49\%) than GMPs (20.17\%) routinely examined patients' oral mucosa $\left(\chi^{2}=69.245, \mathrm{df}=1, \mathrm{p}<0.001\right)$. Of those practitioners who did not routinely examine patients' oral mucosa, one GDP and 155 (65.1\%) GMPs did not examine the oral mucosa of high risk patients.

Question 3, 'What would you consider as risk factors for oral cancer?', was asked as an open question rather than providing the practitioners with the answers and tick boxes. As this was an open question, a wide range of responses was generated. Therefore responses in relation to diet factors and dental factors are reported as merged groups of responses. The risk factors accepted and the grouping of diet and dental factors are shown in Table 1.

GDPs identified a greater number of risk factors (mean 2.62) than GMPs (mean 2.00) $(t=1.969$, df $=257, p<0.0005)$. The distribution of risk factors identified is shown in Figure 2. Smoking was identified well by GMPs and GDPs, however significantly less GMPs (43.28\%) than GDPs (87.22\%) identified alcohol as a risk factor $\left(\chi^{2}=44.448, \mathrm{df}=1, \mathrm{p}<0.001\right)$.

Significantly more GDPs than GMPs reported regularly advising their patients on the risk factors for oral cancer $\left(\chi^{2}=5.196, \mathrm{df}\right.$ $=1, p<0.05)$ and GDPs felt more confident about diagnosing oral cancer from clinical appearance $(\chi=17.863, \mathrm{df}=3, \mathrm{p}<0.001)$.

Question 6, 'What changes within the mouth would you associate with oral cancer?', was again asked as an open question rather than providing the practitioners with the answers and tick boxes. Again, as this was an open question, a wide range of responses was generated. Therefore responses in relation to exophytosis are reported as a merged group of responses. The oral changes accepted and the exophytosis grouping are shown in Table 2. GDPs identified a greater number of oral changes (mean 2.75) than GMPs (mean 1.75) $(\mathrm{t}=1.651$, df $=235, \mathrm{p}$ $<0.0005)$. The distribution of oral changes identified is shown in Figure 3. Significantly fewer GMPs identified erythroplakia, leukoplakia and erythroleukoplakia as oral changes associated with oral cancer.

The vast majority of both GMPs and GDPs selected oral medicine and oral and maxillofacial surgery as their preferred points of referral. GDPs selected oral medicine more often than oral and maxillofacial surgery as their preferred point of referral.

Significantly fewer GMPs felt that they had sufficient knowledge regarding prevention and detection of oral cancer (GMPs 25.2\% and GDPs 54.1\%, $\left.\chi^{2}=10.922, \mathrm{df}=1, \mathrm{p}<0.001\right) .80 .4 \%$ and 71.4\% of GDPs and GMPs respectively requested further information/training on oral cancer with an information pack as the preferred format compared to meetings or seminars.

\section{DISCUSSION}

Unsurprisingly, significantly more GDPs than GMPs routinely examine the oral mucosa of patients. GMPs are less likely to

\begin{tabular}{|l|}
\hline Table 1 Risk factors for oral cancer \\
\hline Tobacco smoking \\
\hline Smokeless tobacco use \\
\hline Betel quid chewing \\
\hline Alcohol consumption \\
\hline UV light exposure \\
\hline Viral factors \\
\hline Immunosuppression \\
\hline Chronic infection \\
\hline Occupation \\
\hline Dietary factors: \\
\hline Diet low in iron \\
\hline Diet low in vitamin A \\
\hline Diet low in vitamin C \\
\hline High fat diet \\
\hline Dental factors: \\
\hline Chronic irritation from jagged teeth \\
\hline $\begin{array}{l}\text { 'Poor dental condition' (poor oral hygiene/number of missing } \\
\text { teeth } \geq 11 \text { ) }\end{array}$ \\
\hline
\end{tabular}

\begin{tabular}{l} 
Table 2 Oral changes associated with oral cancer \\
\hline Ulceration \\
\hline Erythroplakia \\
\hline Leukoplakia \\
\hline Erythroleukoplakia \\
\hline Induration \\
\hline Fixation \\
\hline Bleeding \\
\hline Exophytosis: \\
\hline Mass \\
\hline Lump \\
\hline Growth \\
\hline Necrosis \\
\hline Lymphadenopathy \\
\hline
\end{tabular}

examine a patients' oral mucosa routinely but may do so in relation to the context of the consultation, for example presentation with an oral symptom. ${ }^{20}$ GMPs are more likely to see elderly patients and those at higher risk of oral cancer, ${ }^{18,19}$ however $65.1 \%$ of GMPs responded that they did not screen the oral mucosa of high risk patients. This may be due to a lack of knowledge and confidence in recognising the significance of oral mucosal changes.

Smoking tobacco as a risk factor for oral cancer was identified well by both GMPs and GDPs, whereas 43.3\% of GMPs 
compared with $87.2 \%$ of GDPs identified alcohol as a risk factor. This is consistent with previous studies. ${ }^{15,20,21}$ Thus, in future training of healthcare practitioners, alcohol use must be emphasised as a risk factor for oral cancer. Smoking tobacco and alcohol consumption are associated with 75\% of oral cancers and are the main risk factors in the United Kingdom. Knowledge of other risk factors was poor in both GMPs and GDPs, although some risk factors have less importance, for example missing teeth and chronic irritation from jagged teeth, which are unlikely to be risk factors in isolation. Recent research by Salaspuro and colleagues ${ }^{22}$ has provided some evidence for a potential link between alcohol, poor oral hygiene and oral cancer. They found that some oral bacteria (including various strains of the viridans group of Streptococcus) are able to metabolise ethanol to acetaldehyde (a known carcinogen and mutagen). ${ }^{22}$

More GDPs reported regularly advising patients about risk factors for oral cancer, which may be related to more extensive risk factor knowledge. However, what they say they do and what is actually done may be inconsistent and is not supported in previous studies. ${ }^{20}$ GMPs may provide or refer to smoking cessation services and alcohol support groups more regularly than GDPs, in relation to prevention of coronary heart disease as well as prevention of malignant diseases, and are thus in a better position to offer advice on oral cancer risk factors.

GDPs felt more confident about diagnosing oral cancer from clinical appearance than GMPs. Again, this may be related to a greater knowledge of oral changes associated with oral cancer. Similarly to previous studies, ${ }^{15,20}$ ulceration and leukoplakia were identified more commonly than erythroplakia or erythroleukoplakia by both GMPs and GDPs, despite the greater malignant potential of the latter lesions. Histopathologically, it has been documented that in homogenous erythroplakia 51\% showed invasive carcinoma ${ }^{23}$ and the malignant transformation rate of erythroplakia and erythroleukoplakia can be at least 50\%. ${ }^{24}$ Therefore the significance of erythroplakia and erythroleukoplakia needs to be emphasised in future training of health professionals. Whilst a recent paper found that approximately one in four people were aware of red patches (erythroplakia) as an early sign of oral cancer, they were given a list of conditions from which to choose (or guess). ${ }^{9}$ In our study the question was open - and less than $5 \%$ of GMPs knew that a red patch (erythroplakia) was an early sign of mouth cancer. Open questions test the individual's knowledge when answering rather than selecting from a list of provided answers, which produces a more accurate reflection of the individual's knowledge than their ability to guess correctly. In addition, open questions have also been shown to increase the range of responses and response rates. ${ }^{25,26}$ Some cancers may be silent and the lack of symptoms may contribute to late presentation. ${ }^{27}$ Thus initial symptoms may not reliably predict early disease. Improved GMP, GDP and public knowledge of early signs of oral cancer may therefore lead to earlier presentation.

Oral and maxillofacial surgery and oral medicine departments were the most commonly selected points of referral for patients with a suspected oral cancer. The availability of Dundee Dental Hospital within Tayside may explain why a greater proportion of GDPs selected oral medicine. This may

\section{Fig. 1 Questionnaire}

Questionnaire for general medical/dental practitioners

This questionnaire has been designed with the purpose of using the information to consider ways of improving prevention, early detection and referral of oral cancer by doctors and dentists.

\begin{tabular}{l|l}
\hline Age $(. . . \ldots \ldots \ldots . . . . . . . y r s)$ & Sex - Male/female
\end{tabular}

What year did you first qualify?

Qualifications:

Questions (please circle as appropriate)

1. Do you examine patients' oral mucosa routinely? YES or NO

2. If your answer is no to question 1., do you screen the oral mucosa if the patients are in high risk categories?

YES or NO

3. What would you consider as risk factors for oral cancer?

4. Do you regularly advise patients about the risk factors for oral cancer? YES or NO

5. As regards diagnosing oral cancer from clinical appearance, do you feel? Veryconfident Confident Unsure Very unsure

6. What changes within the mouth would you associate with oral cancer?

7. Where would you refer a patient if you suspected an oral malignancy? (circle one or more)

Plastic surgery ENT Oral and maxillofacial surgery Oral medicine Dentist General practitioner Other

8. Do you feel that you have sufficient knowledge concerning prevention and detection of oral cancer?

YES or NO

9. Would you like more information or training on oral cancer? YES or NO

10. If so which format would you prefer? (tick one or more) Information pack

Lunchtime meeting

Evening seminar

Your answers will be treated as strictly confidential THANK YOU FOR YOUR COOPERATION

not be generalisable to other regions without a dental hospital. There is also the possibility that GMPs and GDPs were steered towards referring to oral medicine and oral and maxillofacial surgery units by virtue of the word 'oral' appearing in the title. In retrospect, bias may have been reduced if this question was left open to the practitioner rather than presented as a closed question.

Fewer GMPs felt they had sufficient knowledge regarding oral cancer prevention and detection. This has also been reflected in previous studies, where confidence about oral cancer knowledge was attributed to a lack of training. ${ }^{20}$ The majority of GMPs and GDPs requested further training in oral cancer prevention and detection. This is hardly surprising given that most generalists would argue that they were not experts 


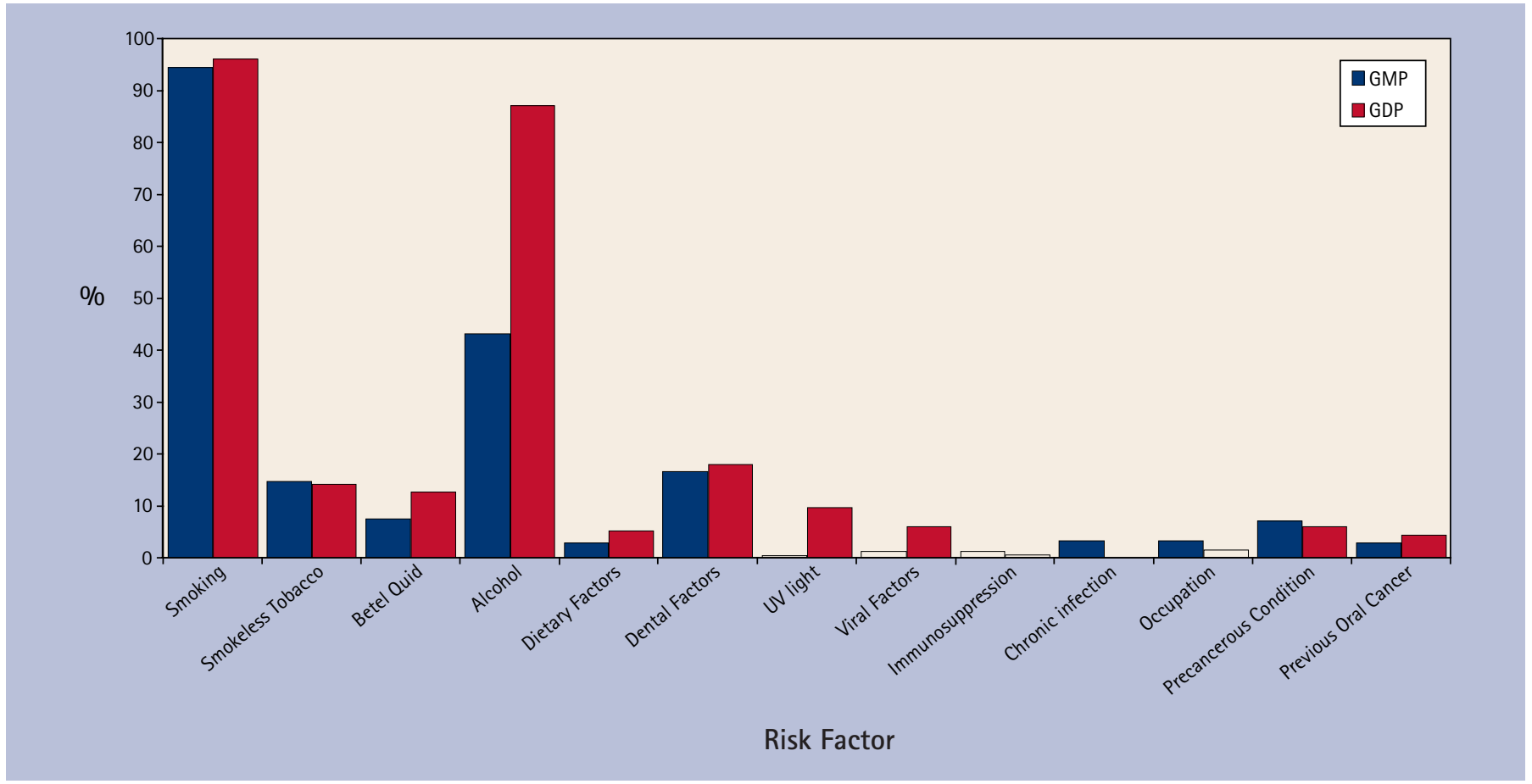

Fig. 2 Distribution of risk factors identified

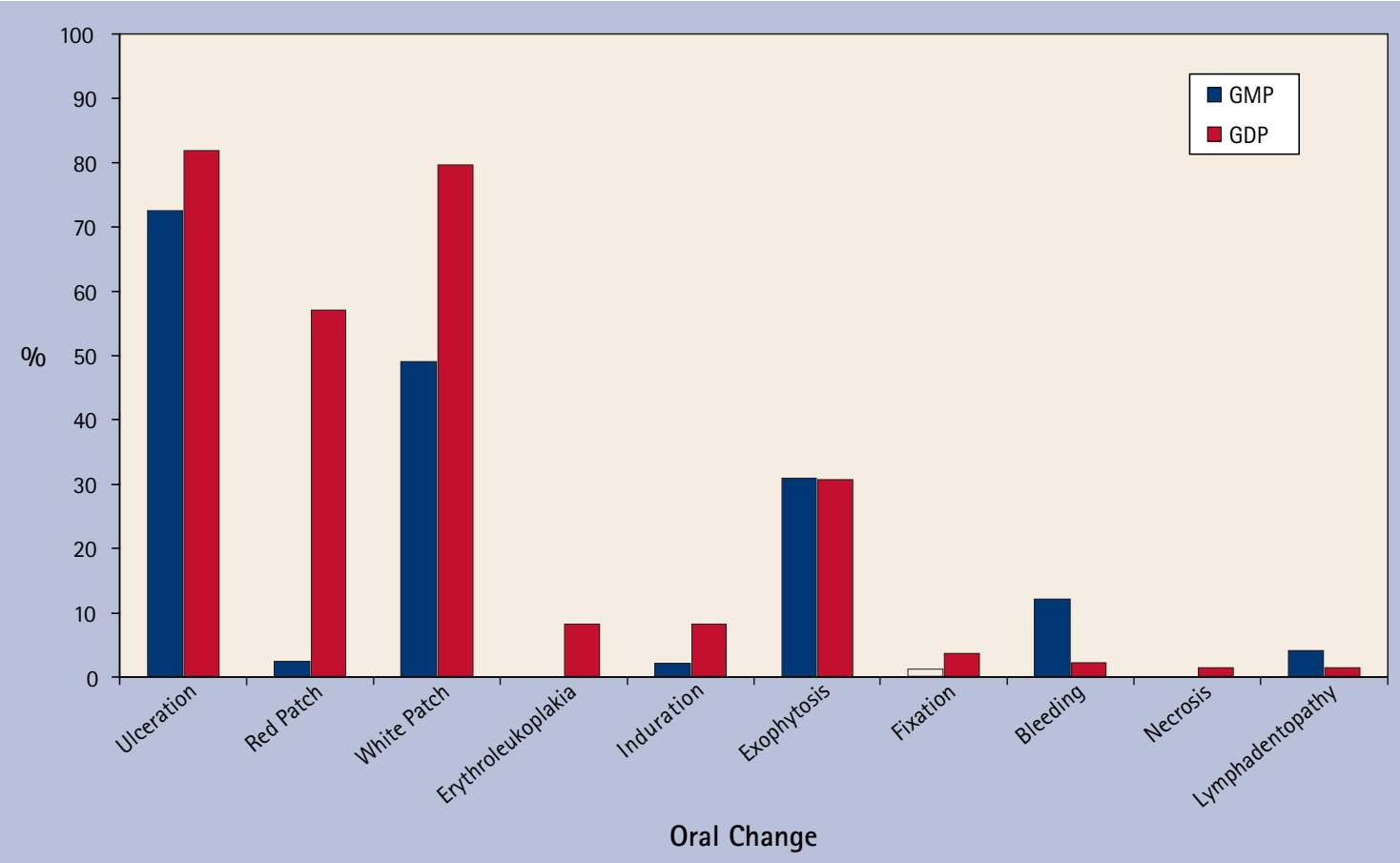

Fig. 3 Distribution of oral changes identified

and therefore might benefit from further information. Recent initiatives in Scotland have been undertaken to raise both public and GMP/GDP cancer awareness via various media. ${ }^{28}$

GMPs have less risk factor knowledge, less knowledge of oral changes associated with oral cancer and are less likely to examine the oral mucosa of patients, including those at high risk of oral cancer, than GDPs. However, patients with oral lesions often present to a GMP. ${ }^{17}$ The cost of an examination by a dentist may prohibit some patients from attending their GDP, ${ }^{20,29}$ although this has been phased out in Wales and is due to be abolished in Scotland. In some individuals, attending a dentist may be associated with bad or unpleasant experiences in the past and this would make them less likely to present to a dentist with oral symptoms. Despite an increase within younger patients, oral cancer is more prevalent in the elderly. Haughney et al. showed that elderly patients were less likely to present for regular dental check-ups and that many patients over 75 years of age, most of whom were edentulous, refused to present to a dentist for examination. Haughney et al. also showed that smokers were less likely to present for regular dental check-ups. ${ }^{30}$ However, these groups of patients may present more regularly to GMPs. ${ }^{18,19}$

Oral cancer awareness of GMPs could be improved by the inclusion of teaching on oral health and oral diseases in the medical 
undergraduate curriculum. A more proactive approach from oral and maxillofacial surgeons and oral physicians towards teaching medical undergraduates should be undertaken. The medical undergraduate curriculum at the University of Dundee benefits from the inclusion of teaching on oral diseases by oral surgeons as part of the gastrointestinal system teaching, and has been favourably received by the students. ${ }^{31}$ Foundation year jobs in oral and maxillofacial surgery for medical graduates pursuing a career in general practice in addition to those pursuing a career in surgery would also increase the level of oral cancer awareness of medical practitioners. ${ }^{32,33}$ Routine examination of the mouths of every patient by general medical practitioners would be unlikely unless this was included as part of general screening examinations, perhaps driven by national guidelines with associated financial incentives. In addition Haughney et al. showed that integration of primary care dental and medical services in the same premises with joint consultation and examination of selected patients increased the oral health awareness of the primary care medical and nursing team. ${ }^{30}$

As the incidence of oral cancer continues to rise, ${ }^{1,2,9}$ the role that general medical (and dental) practitioners may play in prevention and detection of oral cancer assumes ever greater importance. Since approximately 75\% of oral cancer arises in association with alcohol and tobacco, GMPs, who see more patients with alcohol-related diseases, may be better placed to identify patients at such risk. Furthermore, this study highlights the need to address the educational needs of general medical (and dental) practitioners on oral cancer.

1. Conway D I, Stockton D L, Warnakulsuriya K A A S, Ogden G R, Macpherson L M D. Incidence of oral and oropharyngeal cancer in United Kingdom (1990-1999) - recent trends in regional variation. Oral Oncol 2006; 42: 586-592.

2. Moore S R, Johnson N W, Pierce A M, Wilson D F. The epidemiology of mouth cancer: a review of global incidence. Oral Dis 2000; 6: 65-74.

3. Macfarlane G J, Sharp L, Porter S, Franceschi S. Trends in survival from cancers of the oral cavity and pharynx in Scotland: a clue as to why the disease is becoming more common? Br J Cancer 1996; 73: 805-808.

4. Cancer Research UK Open Up to Mouth Cancer website. www.openuptomouthcancer.org. Cancer Research UK, 2005.

5. Hindle I, Nally F. Oral cancer: a comparative study between 1962-67 and 198084 in England and Wales. Br Dent J 1991; 170: 15-20.

6. Johnson N W, Warnakulsuriya K A A S. Epidemiology and aetiology of oral cancer in the united kingdom. Community Dent Health 1993; 10 (Suppl 1): 13-29.

7. Speight P M, Morgan P R. The natural history of oral cancer and precancer. Community Dent Health 1993: 10 (Suppl 1): 31-41.

8. Ogden G R, Graham C. Mouth cancer awareness in Dundee, Scotland. Public perception and attitudes. UICC Delhi conference proceedings. ALC Macmillan, 2003.
9. West R, AlKhatib M N, McNeill A, Bedi R. Awareness of mouth cancer in Great Britain. Br Dent J 2006; 200: 167-169.

10. McLeod N M H, Saeed N R, Ali E A. Oral cancer: delays in referral and diagnosis persist. Br Dent J 2005; 198: 681-684.

11. Warnakulsuriya K A A S, Harris C K, Scarrott D M et al. An alarming lack of public awareness towards oral cancer. Br Dent J 1999; 187: 319-322.

12. Boyle P, Macfarlane G J, Scully C. Oral cancer: necessity for prevention strategies. Lancet 1993; 342: 1129 .

13. Hollows $P, M c$ Andrew P G, Perini M G. Delays in the referral and treatment of oral squamous cell carcinoma. Br Dent J 2000; 188: 262-265.

14. Schnetler J F. Oral cancer diagnosis and delays in referral. Br J Oral Maxillofac Surg 1992; 30: 210-213

15. Greenwood M, Lowry R J. Primary care clinicians' knowledge of oral cancer: a study of dentists and doctors in the North East of England. Br Dent J 2001: 191: 510-512.

16. Scully C, Malamos D, Levers B G, Porter S, Prime S S. Sources and patterns of referrals of oral cancer: role of general practitioners. Br Med J (Clin Res Ed) 1986; 293: 599-601.

17. Langdon J D. Oral cancer: raising the level of awareness. Dent Update 1995; 22: 269-270.

18. Yellowitz J A, Goodman H S. Assessing physicians' and dentists' oral cancer knowledge, opinions and practices. J Am Dent Assoc 1995; 126: 53-60.

19. Goodman H S, Yellowitz J A, Horowitz A M. Oral cancer prevention. The role of family practitioners. Arch Fam Med 1995; 4: 628-636.

20. Macpherson L M D, McCann M F, Gibson J, Binnie VI, Stephen K W. The role of primary health care professionals in oral cancer prevention and detection. Br Dent J 2003; 195: 277-281.

21. McCunniff M D, Barker G J, Barker B E, Williams K. Professionals' baseline knowledge of oral/pharyngeal cancers. J Cancer Educ 2000; 15: 79-81.

22. Kurkivuori J, Salaspuro V, Kaihovaara P et al. Acetaldehyde production from ethanol by oral streptococci. Oral Oncol 2007; 43: 181-186.

23. Reichart P A. Oral erythroplakia - a review. Oral Oncol 2005; 41: 551-561.

24. Bouquot J E, Ephros H. Erythroplakia: the dangerous red mucosa. Pract Periodontics Aesthet Dent 1995; 7: 59-67.

25. O'Cathain A, Thomas K J. "Any other comments?" Open questions on questionnaires - a bane or a bonus to research. BMC Med Res Methodol 2004; 4: 25.

26. McColl $E_{1}$ Jacoby $A$, Thomas $L$ et al. Design and use of questionnaires: a review of best practice applicable to surveys of health service staff and patients. Health Technol Assess 2001: 5(31): 1-256.

27. McGurk M, Chan C, Jones J, O'Regan E, Sherriff M. Delay in diagnosis and its effect on outcome in head and neck cancer. Br J Oral Maxillofac Surg 2005; 43: 281-284.

28. Macpherson L M D, Gibson J, Binnie V I, Conway D I. Oral cancer prevention and detection for the primary health care team: the oral cancer awareness group, www.gla.ac.uk/schools/dental/oralcancer.

29. Ogden G R, Cowpe J G, Chisholm D M. Cost of oral screening. Lancet 1991; 337: 920-921.

30. Haughney M G J, Devennie J C, MacPherson L M D, Mason D K. Integration of primary care dental and medical services: a three year study. Br Dent J 1998; 184: 343-347.

31. Ogden G R, Ker J. Re: Kissun et al. General medical practitioners' knowledge of the specialty of oral and maxillofacial surgery. Br J Oral Maxillofac Surg 1998; 36: $479-480$.

32. Kissun D, Rogers A, Bastin M, Frame J W. General medical practitioners' knowledge of the specialty of oral and maxillofacial surgery. Br J Oral Maxillofac Surg 1998; 36: 75-76.

33. Ogden G R, Kerr J S, Green M. Utility of interprofessional peer examiners in an objective structured clinical examination (OSCE): can dental students act as examiners? Br Dent J 2000; 189: 162-164. 